\title{
Iodeto de potássio suportado em MCM-41 como catalisador para síntese de biodiesel utilizando micro-ondas
}

Antônio Alex de Lima Silva ( Mestrando em Ciências Naturais do PPGCN na Universidade do Estado do RN - UERN);

Marilia Gabriela Araújo Pereira (Graduanda em Química na Universidade do Estado do Rio Grande do

$$
\text { Norte-UERN); }
$$

Luiz Di Souza (Prof. Ad. do Dq e do PPGCN na Universidade do Estado do Rio Grande do Norte -

$$
\text { UERN); }
$$

Anne Gabriella Dias Santos Caldeira (Prof ${ }^{a}$. Ad. do Dq e do PPGCN na Universidade do Estado do Rio Grande do Norte - UERN);

*alex_rodrigues15@hotmail.com

\section{Resumo:}

O biodiesel é um biocombustível derivado de biomassa renovável de queima limpa. Atualmente, sua produção ocorre por transesterificação via catálise básica homogênea. Porém a difícil separação do catalisador e a geração de muito efluente são desvantagens dessa rota. Por isso se procuram formas mais limpas para sua produção. Os catalisadores heterogêneos se mostram como alternativa, por oferecer vantagens como: recuperação e reutilização do catalisador e a geração de menos resíduos. O uso de sais e óxidos metálicos impregnados em materiais mesoporosos tem sido um sucesso por aliar a atividade catalítica dos metais a maior área superficial de materiais como o MCM-41. Outra forma de melhorar o processo é fazer a reação usando micro-ondas, que devido a sua capacidade de aquecimento, pode gerar procedimentos rápidos e eficientes. O Iodo na forma de sais $(\mathrm{KI})$, tem obtido resultado na catalise, porém, se faz necessário estudar mais o seu uso na transesterificação. Tendo em vista isso este trabalho tem como objetivo avaliar a atividade do Iodeto de Potássio suportado em MCM-41 na transesterificação com microondas do óleo de girassol. O MCM-41 foi sintetizado e impregnado com Kl nas concentrações de 2,5, 5, 7,5 e 10\% essas amostras foram caracterizadas por Difração de raio-X. A transesterificação foi realizada usando de micro-ondas e a conversão foi avaliada por termogravimetria. Os resultados mostraram que o lodeto de potássio apresentou atividade catalítica em todas as concentrações utilizadas, porem a porcentagem que melhor se comportou na reação foi 7,5 \% Kl em MCM-41, que apresentou uma conversão de $82 \%$. O Uso do micro ondas se mostrou promissor, visto que o biodiesel foi obtido de forma rápida e com boa conversão.

\section{Palavras-chave:}

Girassol; Biodiesel; Ecodiesel 


\section{1- I NTRODUÇÃO}

A proteção ao meio ambiente e a utilização sensata dos seus recursos naturais são requisitos muito importantes tanto para o bem do planeta como de seus habitantes. Porém o uso indevido desses recursos vem acontecendo vários impactos socioeconômicos e ambientais ao longo das últimas décadas, principalmente em relação às fontes energéticas de origem fóssil. Em função dessa problemática, surgiu a necessidade da utilização de alternativas energéticas que possam diminuir a dependência do petróleo e seus derivados, e paralelamente, diminuam os impactos ocasionados por essa utilização (MEDEIROS, 2013). Neste sentido a utilização da biomassa na produção de biocombustíveis se mostra como opção, principalmente no biodiesel.

O Biodisel é definido pela Agencia Nacional de Petróleo, Gás Natural e Biocombustível (ANP) como um biocombustível derivado de biomassa renovável para uso em motores a combustão interna com ignição por compressão ou, conforme regulamentos para outro tipo de geração de energia, que possa substituir parcial ou totalmente combustíveis de origem fóssil (ANP, 2008). Estudos realizados em biodiesel mostram que a utilização do mesmo ou de misturas deste com o diesel mineral apresentaram algumas vantagens e desvantagens (KEGL, 2008; MACLEOD et al, 2008; LAPUERTA et al, 2008; SANTOS et al, 2011, SILVA, et al, 2015).

Dentre as principais vantagens podem ser citadas: o consumo equivalente ao diesel, à dispensa qualquer tipo de conversão ao motor, a emissão de $50 \%$ a menos de CO e particulados, $78 \%$ a menos de gases do efeito estufa, o aumento da lubrificação do motor, além de aumentar a vida útil do motor e principalmente o fato de ser um combustível renovável e ambientalmente correto (TAPANES; PEREZ; CRUZ, 2013). Porem alguns desvantagens devem ser destacadas como: a produção grandes volumes de glicerina e água residual por catálise homogênea, produção de energia ligeiramente mais baixa, se comparada a um volume equivalente do diesel, além do fato de ser mais caro que o diesel regular, visto que depende da área para plantação da matéria-prima utilizada (SANTOS et al., 2010).

Com a crescente produção de biodiesel se faz necessário à diversificação das matérias primas na produção do biocombustível. Segundo a ANP no boletim de fevereiro de 2014 maior parte do biodiesel no produzido no Brasil é proveniente da soja. Neste contexto, com o um possível aumento da produção brasileira de biodiesel, surge à necessidade de matérias-primas alternativas começaram a ser utilizados em maior escala. O girassol (helianthus annuus) pode ser utilizado, além ser produzir bastante óleo (BELTRÃO; OLIVEIRA, 2007), a espécie apresenta melhor tolerância à seca do que o milho ou o sorgo, possui baixa incidência de doenças, além dos benefícios que o girassol proporciona às culturas subsequentes. O Plantio da oleaginosa também beneficiaria a agricultura familiar promovendo a inclusão social e o desenvolvimento regional por meio de geração de emprego e renda para os agricultores. (PINTO, 2014)

Atualmente sua produção de biodiesel no Brasil, ocorre através da transesterificação utilizando a catalise básica homogênea, que tem como principais vantagens o alto rendimento e conversão além do baixo tempo de reação (HELWANI, 2009; CORDEIRO et al., 2011). No entanto, esta rota catalítica apresenta alguns inconvenientes, como: a difícil separação do catalisador ao final da reação, produção indesejada de sabões que diminuem o rendimento da reação e dificultam o processo, produção de grandes volumes glicerina, que geralmente não é purificado ao final da reação (GALLO, 2005; GALVÃO, 2012), além de produzir muita água residual geralmente não possui um destino correto, visto que não são encontrados trabalhos na literatura sobre o destino final desta água (GRANGEIRO, 2009). Por isso diversos pesquisadores vêm estudando formas mais rápidas, limpas, eficientes e seguras, para produção de biodiesel. Com isso os catalisadores heterogêneos têm atraído um grande interesse das áreas científicas e industriais, pois oferecem vantagens como: fácil manuseio, recuperação e reutilização do catalisador, além de gerar menos resíduos (SOLDI et al., 2009, GHESTI, 2012).

Vários catalisadores heterogêneos básicos e ácidos foram testados na reação de transesterificação de triglicerídeos com metanol (SERIO et al, 2006; GARCIA et al., 2008). Os catalisadores básicos apresentaram taxas de conversões maiores do que os sólidos ácidos (TAPANES, 2008, VENKAT REDDY; OSHEL; VERKADE, 2009; TAPANES, PEREZ, CRUZ, 2013). Nesta classe encontram-se, óxidos, hidróxidos e alcóxidos de metais alcalinos 
terrosos. Em contra partida, a aplicação de tais compostos na síntese de biodiesel apresenta problemas devido à fácil carbonatação e lixiviação parcial dos mesmos no meio reacional (GALVÃO et al, 2012). Uma das maneiras de minimizar esses tipos de problemas tais incorporar o óxido em um suporte catalítico.

A peneira molecular mesoporosa do tipo MCM-41, se apresenta como um suporte catalítico favorável para o desenvolvimento de novos catalisadores, com fases ativas e bem dispersas. Ela apresenta um arranjo hexagonal de mesoporos unidimensionais com diâmetro que pode variar entre 2-10 nm, alta estabilidade térmica, volume de poros alto e área superficial acima de $700 \mathrm{~m}^{2} \mathrm{~g}^{-1}$, além da acessibilidade de moléculas grandes (triglicerídeos e ácidos graxos) (CASTRO, 2010). Estas características toranm o MCM-41 um material bastante promissor na catálise, servindo como suporte para catalisadores heterogêneos, oferecendo novos caminhos para a síntese.

Trabalhos com a utilização de Iodo e seus sais têm sido relatados na literatura (RAMALINGA et al, 2002; LI E XIE, 2006; SOUZA et al, 2010; ARAÚJO et al, 2011, GALVÃO, et al, 2012). No entanto, os trabalhos ainda têm necessidade de otimização, devido ao longo tempo de reação (em media 24 h) e a não recuperação do Iodo ao fim do experimento, para se tornar economicamente viável. Nesta perspectiva pode se impregnar o iodo ou sais a base de Iodo, em suportes mesoposoros, a fim de avaliar sua atividade catalítica nas reações de forma heterogênea. GALVÃO et al, (2012), realizou um trabalho em que iodeto de potássio é suportado em peneiras moleculares mesoporosas (SBA-15 E MCM-41) como catalisador básico para síntese de biodiesel onde as taxas de conversões são consideráveis.

Outra alternativa de melhorar as condições da síntese, é uso de técnicas instrumentais apropriadas as reações de transesterificação como o aquecimento por micro-ondas. Essa técnica tem como principais vantagens em relação ao aquecimento convencional: as taxas de aquecimento maiores que no aquecimento convencional, a energia é transferida diretamente para a amostra, não havendo contato físico com a fonte de aquecimento e possibilidade de maiores rendimentos, maior seletividade e menor decomposição térmica, tanto em sistemas homogêneos como heterogêneos (SANSEVERINO, 2002; FILHO, 2009; MOURA, 2010). Por tais fatos o uso do micro-ondas, vem sendo utilizado como uma alternativa promissora para produção de biodiesel, pois, acelera a reação, diminui o tempo, aumenta o rendimento e conversão, além de permitir a execução de modo simples, eficiente e seguro como é mostrado por FILHO, (2009), MOURA, (2010), e GAMA, et al, (2014). Tendo em vista isso este trabalho tem como objetivo avaliar a atividade do Iodeto de Potássio suportado em MCM-41 na transesterificação com microondas do óleo de girassol.

\section{2- Materiais e métodos}

\subsection{Síntese do MCM-41}

A síntese do catalisador foi realizada utilizando a proporção entre os reagentes de 1,0 CTMABr. 1,0 NaOH. 4,0 $\mathrm{SiO}_{2}$. 200,0 $\mathrm{H}_{2} \mathrm{O}$. Primeiramente, a fonte de sílica e hidróxido de sódio foi adicionado a metade da água destilada necessária, deixando em agitação magnética por $2 \mathrm{~h}$ a 60 ${ }^{\circ} \mathrm{C}$. Em seguida, foi feita uma segunda solução pela mistura do direcionador e a outra metade de água, que também permaneceu sob agitação por 30 minutos em temperatura ambiente. Decorrido o tempo necessário, a segunda solução foi adicionada a primeira, permanecendo sob agitação por mais 30 minutos. O gel de síntese obtido foi transferido para uma autoclave de teflon e o conjunto levado a uma estufa a $100{ }^{\circ} \mathrm{C}$, permanecendo por um período de 5 dias, em que realizou-se correções do $\mathrm{pH}$ do gel para uma faixa entre 9-10. Para isto, utilizou-se uma solução de ácido acético a $30 \%$. Por fim, o material foi filtrado à vácuo, lavado com água destilada, seco em estufa a $100{ }^{\circ} \mathrm{C}$ durante 4 h e submetido a calcinação. Para a calcinação, o material foi aquecido em uma razão de aquecimento de $10^{\circ} \mathrm{C} \cdot \mathrm{min}^{-1}$ até $550{ }^{\circ} \mathrm{C}$ e mantido nesta temperatura durante $2 \mathrm{~h}$ sob fluxo ar sintético a $100 \mathrm{~mL} \cdot \mathrm{min}^{-1}$. (BARBOSA, 2009, CASTRO, 2010).

\subsection{I mpregnações do material}

As impregnações foram feitas com diferentes concentrações de KI sendo elas: 2,5\%, 5\%, 7,5\% e $10 \%$. Estes suportes foram impregnados pelo método via excesso de solvente com soluções 
aquosas de iodeto de potássio (GALVÃO, 2012; SHIMABUKU, 2013). Para tanto, $50 \mathrm{~mL}$ das soluções diluídas de iodeto de potássio, nas respectivas \% em peso, foram misturados a 1,0 g de suporte. Em seguida, esse sistema teve sua água reduzida por meio de agitação, a $80{ }^{\circ} \mathrm{C}$, sob pressão ambiente durante 30 minutos. Em seguida o material foi seco em estufa a $100{ }^{\circ} \mathrm{C}$. Posteriormente o sólido impregnado foi calcinado a $500{ }^{\circ} \mathrm{C}$ sob fluxo de ar $(100 \mathrm{~mL}$ min-1) por 3 h (GALVÃO, et al, 2012).

\subsection{Difração de raios-X}

Para confirmar se a estrutura típica dos materiais MCM-41, bem como alteração das estruturas causadas pela incorporação do KI foi a técnica de difração de Raios- X (DRX). As amostras foram analisadas utilizando o equipamento Rigaku modelo Mini Flex II, usando radiações de $\mathrm{CuK} \alpha$, em uma voltagem de $30 \mathrm{k} / \mathrm{V}$ e corrente de tubo de $30 \mathrm{~m}$, as amostras foram realizadas em baixo ângulo (20) de $1^{\circ}$ a $10,0^{\circ}$.

\subsection{Reações de transesterificação}

As reações de transesterificação foram realizadas em um balão adaptado a um sistema de refluxo e aquecido um forno de Microondas com potência a de $500 \mathrm{~W}$ a uma temperatura de $65^{\circ} \mathrm{C}$, durante 4 minutos, na reação foi utilizada uma razão molar 15:1 álcool/óleo $(\mathrm{m} / \mathrm{m})$ e 3\% em massa dos catalisadores sintetizados. (GALVÃO et al, 2012). A fim de analisar as conversões foram realizadas analises de TG.

\subsection{Termogravimetria (TG/ DTG)}

A análise termogravimétrica (TG/DTG) foi realizada com o intuito, principal, de acompanhar as perdas de massa das amostras e estimar a conversão de óleo de girassol em ésteres metílicos. Para isso em torno de $5 \mathrm{mg}$ das amostras foram submetidas a uma faixa de 30 a $800^{\circ} \mathrm{C}$. Para o processo foi utilizado um cadinho de alumina em uma razão de aquecimento de $10^{\circ} / \mathrm{min}$, sob atmosfera de nitrogênio com fluxo de aproximadamente $70 \mathrm{~mL} \cdot \mathrm{min}^{-1}$. Todas as analises foram realizadas em um equipamento STA 449 jupiter da Netzsch. (SANTOS, 2010; OLIVEIRA, et al, 2012; SILVA, et al 2015).

\section{3- RESULTADOS E DISCUSSÃO}

Após a síntese e calcinação dos materiais realizou-se a analise de difração de raio X. Esta técnica permite verificar se a estrutura hexagonal mesoporosa do MCM-41 foi formada após a síntese e calcinação dos materiais. Segundo BECK et al. (1991), o difratograma padrão do MCM-41 apresenta de três a cinco picos de reflexão dos planos (100), (110), (200), (210) e (300). Mesmo com a presença de apenas um pico de reflexão do plano (100) já indica a formação da estrutura mesoporosa, mesmo que de forma desordenada. No entanto, a presença dos cinco picos nos fornece a obtenção de um material de alta qualidade e de estrutura bem definida de mesoporos. Para o material sintetizado na forma pura (sem KI), conforme mostrado no gráfico 1, todos os picos de difração característicos da estrutura hexagonal mesoporosa do MCM-41 aparecem no difratograma, o que indica que a estrutura do material foi formada e se encontra bem organizada. 


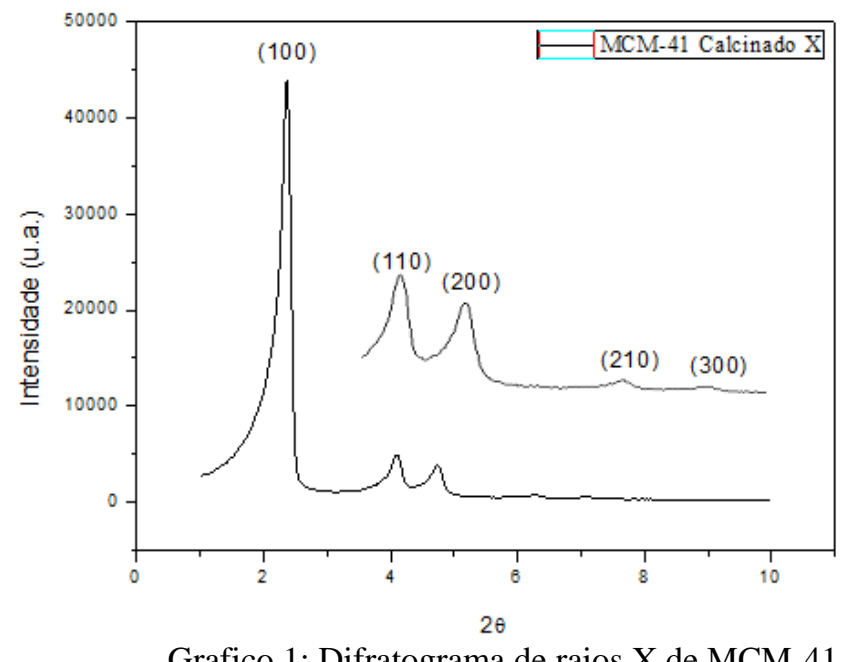

Depois de confirmada a formação da estrutura do MCM-41, realizou-se a impregnações em diferentes concentrações de Iodeto de Potássio (KI), mesmo com a impregnação do material o difratograma mostrado no gráfico 2, indica que ouve uma desorganização, mais o pico de difração 100 foi conservado. O que evidencia que mesmo após a impregnação do KI a estrutura não foi destruída.

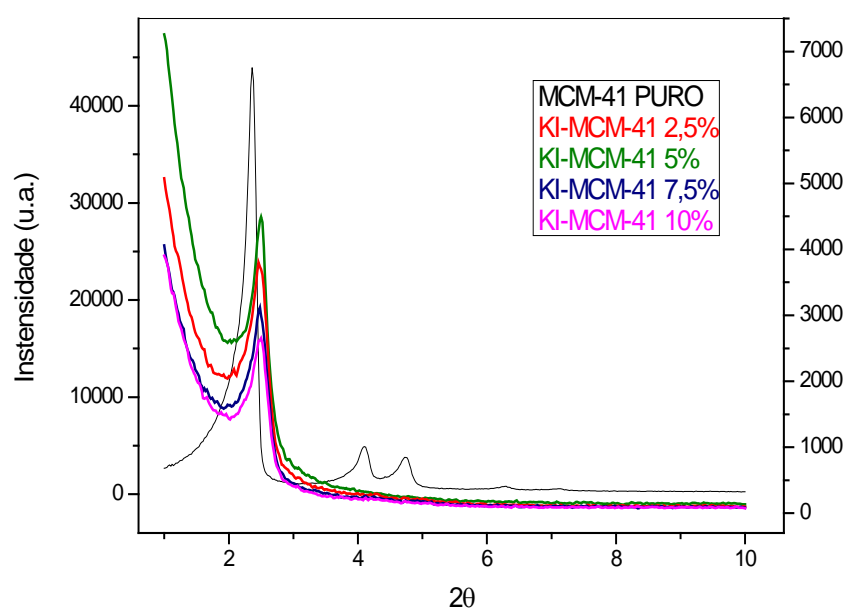

Gráfico 2- Difratogramas de raios X do MCM-4 puro e para amostras impregnadas

Após as impregnações as reações foram realizas do micro-ondas. As amostras apresentaram comportamento semelhante na analise termogravimétrica e no gráfico 3 é apresentado o melhor resultado para a reação de transesterificação. Com base nos resultados obtidos na análise termogravimétrica o catalisador apresentou melhor atividade foi o que possui $7.5 \%$ de KI que apresentou $82 \%$ de conversão. O resultado indica que nesta concentração pode ter ocorrido uma melhor interação sal e o suporte, podendo ter resultado em uma maior quantidade sítios básicos na superfície e no interior dos poros o que contribuiu para a conversão.

Os resultados de conversão foram avaliados com base na diminuição da temperatura de volatilização do óleo em comparação com as amostras obtidas com os diferentes catalisadores. Devido a analise termogravimétrica (TG) não ser a técnica padrão para avaliar a conversão, se faz necessário outras técnicas, como infravermelho, por exemplo, para confirmar a formação dos ésteres metílicos.

Um fato que cabe destaque é o tempo de obtenção do biodiesel, que foi de apenas 4 minutos, esse tempo é muito inferior ao tempo utilizado pelo processo tradicional que gira em torno de 2 horas de reação. O tempo é um fator primordial quando se pensa em escala industrial 
visto que um baixo tempo de reação implica na economia de energia, bem como a uma maior produção em pouco tempo.

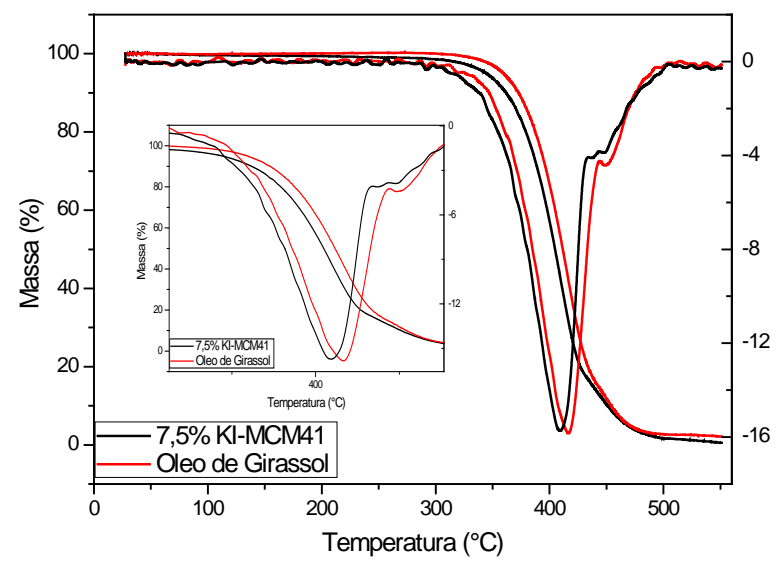

Gráfico 3- Curvas TG/DTG do biodiesel utilizando 7,5\% KI-MCM 41 e óleo de Girassol

\title{
4- CONCLUSÕES
}

O Iodeto de potássio apresentou atividade catalítica em todas as concentrações utilizadas no suporte, porem a porcentagem que melhor se comportou na reação foi 7,5 \% em MCM-41 onde apresentou uma conversão de óleo em biodiesel, 82\%. O Uso do micro ondas associado a catalise heterogênea se mostrou promissor, visto que o biodiesel foi obtido de forma rápida e com boa conversão. Todavia faz necessário a realização de mais testes a fim de otimizar as condições da reação.

\section{Potassium iodide supported on MCM- 41 as catalyst for biodiesel synthesis using microwave}

\begin{abstract}
Biodiesel is a biofuel derived from renewable biomass for clean burning. Currently, its production takes place by transesterification via homogeneous base catalysis. But the difficult separation of the catalyst and the generation of much effluent are disadvantages of this route. So if you seek cleaner ways to produce it. Heterogeneous catalysts are shown as an alternative, by offering advantages such as: recovery and reuse of the catalyst and the generation of less waste. The use of salts and impregnated metal oxides in mesoporous materials has been successful for combining the catalytic activity of metals on higher surface area materials such as MCM-41. Another way to improve the process is to make the reaction using microwave, which due to its heating capacity, can generate quick and efficient procedures. Iodine in the form of salts (KI) result is obtained in catalysis, however, it is more necessary study their use in transesterification. Considering that this study aims to evaluate the activity of potassium iodide supported on MCM-41 in the transesterification with sunflower oil microwave. MCM-41 was synthesized and impregnated with $\mathrm{KI}$ in concentrations of 2.5, 5, 7.5 and $10 \%$ of these samples were characterized by X-ray diffraction. The transesterification was carried out using microwave and the conversion was measured by thermogravimetric analysis. The results showed that potassium iodide showed catalytic activity at all concentrations used, however the percentage best behaved in reaction was $7.5 \% \mathrm{KI}$ in MCM-41, which showed a $82 \%$ conversion. The use of microwave is promising, as biodiesel was obtained quickly and in good conversion.
\end{abstract}

Keywords: Microwave; transesterification; heterogeneous catalysis. 


\section{REFERENCIAS BIBLIOGRÁFI CAS}

AGÊNCIA NACIONAL DE PETRÓLEO, GÁS NATURAL E BIOCOMBUSTÍVEIS. RESOLUÇÂO no 7, de 19/03/2008 Disponível em: < http://www.anp.gov.br/biocombustiveis/biodiesel.asp>. Acesso em: 25 de J aneiro. 2014.

ARAUJ O, Aruzza Mabel. Morais et al, Química no Brasil, V. 4, №.1, 2011.

BECK, J effrey S., Method for synthesizing mesoporous crystalline material. US Patent 5, v. 57, p.296, 1991.

BELTRÃO; Napoleão Esberard de Macêdo; OLIVEIRA; Maria Isaura Pereira, Oleaginosas potencias do nordeste para produção de biodiesel, Documentos MAPA, N.177, Campina Grande - PB, 2007.

CORDEIRO, Claudiney Soares et al. Catalisadores heterogêneos para a produção de monoésteres graxos (biodiesel) Química Nova, V. 34, №. 3, 2011.

FILHO, Agustinho Amâncio da Silva, Produção de biodiesel pela transesterificação alcalina homogênea do óleo de soja com metanol utilizando irradiação de micro-ondas, mestrado, Universidade Federal Rural do Rio de J aneiro, 2009.

GALLO, Jean Marcel Ribeiro, Síntese e caracterização de [Nb]-MCM-41 e NbxOy(OH)zMontmorilonita e aplicações em catalise redox e ácida, Dissertação de mestrado, UNICAMP, 2005.

GALVÃO, Luzia Patrícia Fernandes de Carvalho et al. Iodeto de potássio suportado em peneiras moleculares mesoporosas (SBA-15 e MCM-41) como catalisador básico para síntese de biodiesel, Química Nova, V. 35, N. 1, 2012.

GARCIA, Camica Martins. et al., Transesterification of soybean oil catalyzed bysulfated zirconia, Bio resource Technol., V. 99, №.1 2008

GHESTI, Grace F et al. Produção de biodiesel via transesterificação etílica com zeólitas básicas, Química Nova, V. 35, №. 1, 2012.

GRANGEI RO, Rosa Virgínia Tavares, Caracterização da água de lavagem proveniente da purificação do biodiesel, Mestrado em química, UFPB, 2009.

HELWANI Z et al. Solid heterogeneous catalysts for transesterification of triglycerides with, Applied Catalysis, A. General, V. 363, 2009.

KEGL, B. Effects biodiesel on emissions of a bus diesel engine, Bio resource Technology. V. 88, 2008

LAPUERTA, Magin; RODRIGUEZ-FERNANDEZ, José.; AGUDELO, John. R. Diesel particulate emissions from used cooking oil biodiesel, Bio resource Technology, V. 99, 2008.

$\mathrm{LI}, \mathrm{H}$;; XIE, W. Transesterification of soybean oil to Biodiesel with $\mathrm{Zn} / 12$ catalyst, Catalysis Letters. V. 107, n. 1-2, 2006.

MACLEOD, Clarie. S. et al, Evaluation of the activity and stability of alkali-doped metal oxide catalysts for application to an intensified method of biodiesel production, Chemical engineering J ournal, V. 135, 2008.

MEDEIROS, Mayara Rayane Biodiesel em Guamaré/RN: estágio inicial de implantação, 62 (Graduação em Ciência e Tecnologia) Universidade Federal Rural do Semiárido, Campus Angicos, 62 p. 2013.

MOURA, B. S, Transesterificação Alcalina de Óleos Vegetais para Produção de Biodiesel: Avaliação técnica e econômica, (Mestrado em química) Universidade Federal Rural do Rio de J aneiro, 146 f., 2010.

OLIVEIRA, Daniele da Silva et al, Obtenção do biodiesel através datransesterificação do óleo de moringa oleífera lam, HOLOS, Vol 1, №28, 2012.

PINTO, Vasco de Lima, Efeitos do envelhecimento na degradação termoxidativa do sebo bovino e seus biocombustiveis via analise de UV - VIS, 151p. Dissertação (Mestrado em Ciências Naturais), UERN, Mossoró, 2014

RAMALINGA K.; VIJAYALAKSHMI P.; KAIMAL T. N. B. A mild and efficient method for esterification and transesterification catalyzed by iodine. Tetrahedron letters. vol. $43, \mathrm{n}$. 5, p.879-882, 2002.

SANTOS, A. G. D. Avaliação da estabilidade térmica do biodiesel de algodão, girassol, dendê e sebo bovino. Dissertação (Mestrado em Química), UFRN, Natal, RN 2010.

SANTOS, Anne Gabriella Dias et al, Characterization and kinetic study of sunflower oil and biodiesel, J ournal of Thermal Analysis and Calorimetry, V. 106, 2011. 
SERIO, M. Di; et al., Transesterification of Soybean Oil to Biodiesel by Using Heterogeneous Basic Catalysts I nd. Eng. Chem. Res. Vol. 45, p. 3009, 2006.

SHIMABUKU, Quelen Letícia, Aplicação de carvão ativado impregnado com íons de zinco para remoção de cistos de giardia SPP, e-xacta, V. 6, №. 1, 2013.

SILVA, Antônio Alex de Lima et al, Síntese e Caracterização de Biodiesel de Sebo Bovino e de sua Mistura B10, Orbital: Electron. J. Chem. V.7, 2015.

SOLDI Rafael A. et al. Soybean oil and beef tallow alcoholysis by acid heterogeneous catalysis/ Applied Catalysis A: General V. 361, 2009.

SOUZA, Luiz Di et al., Produção e caracterização físico-química de biodieseis via catalise heterogênea utilizando I odo sublimado, Química no Brasil, V. 4, 2010.

TAPANES, Neyda de la Caridad Om et al, Biodiesel no brasil: matérias primas e tecnologias de produção, AS\&T, V.1, n. 1, 2013.

TAPANES, Neyda de la Caridad Om, Produção de biodiesel a partir da transesterificação de óleo de pinhão manso (jatropha curcas lin): estudo teórico e experimental, tese de doutorado, UFRJ, 2008.

VENKAT REDDY, C. R.; OSHEL, R.; VERKADE, J. G.; Energy Fuels Thermogravimetric Quantification of Biodiesel Produced via Alkali Catalyzed Transesterification of Soybean oil, Energy \& Fuels, V. 23, 2009. 